\title{
Soluble Lie algebras having finite-dimensional maximal subalgebras
}

\section{lan N. Stewart}

\begin{abstract}
Infinite-dimensional soluble Lie algebras can possess maximal subalgebras which are finite-dimensional. We give a fairly complete description of such algebras: over a field of prime characteristic they do not exist; over a field of zero characteristic then, modulo the core of the aforesaid maximal subalgebra, they are split extensions of an abelian minimal ideal by the maximal subalgebra. If the field is algebraically closed, or if the maximal subalgebra is supersoluble, then all finitedimensional maximal subalgebras are conjugate under the group of automorphisms generated by exponentials of inner derivations by elements of the Fitting radical. An example is given to indicate the differences encountered in the insoluble case, and the nonexistence of group-theoretic analogues is briefly discussed.
\end{abstract}

\section{Introduction}

It is possible for an infinite-dimensional soluble Lie algebra to have a finite-dimensional maximal subalgebra. (Here and elsewhere 'infinitedimensional' must be read as 'not finite-dimensional' rather than 'not necessarily finite-dimensional' to exclude trivial cases.) There is an example in Hartley [5] p. 269, which takes the form of a split extension $L=P \ddagger Q$ where $Q$ is finite-dimensional and $P$ is an infinitedimensional irreducible $Q$-module. The main object of this paper is to study the structure of infinite-dimensional soluble Lie algebras which have

Received 11 April 1974. This work was carried out at the Universität Tübingen and financed by the Alexander von Humboldt-Stiftung, to whom the author is grateful for making it possible. 
a finite-dimensional maximal subalgebra. It turns out that such algebras have a structure 'close to' that of Hartley's example. From this follows an a priori unexpected result: in an infinite-dimensional soluble algebra, the finite-dimensional maximal subalgebras are all conjugate, subject to mild restrictions on the field. The proof of this theorem requires some results on split extensions of irreducible modules, which we dispose of in Section 2. In Section 3 we give a reasonably complete description of the algebras referred to in the title (including a proof that over fields of prime characteristic they do not exist) and use this and the results of Section 2 to prove the conjugacy theorem. The final section extends a few results to 'submaximal subalgebras', gives an example to show that the insoluble case is quite different, and discusses possible group-theoretic analogues of the results obtained.

Notation and terminology, now largely standard, will be as in [9], pp. 291-294, or [1]. Additional notation will be defined as required.

\section{Complements to minimal ideals}

Let $L$ be a Lie algebra having an abelian minimal ideal $A$ with a

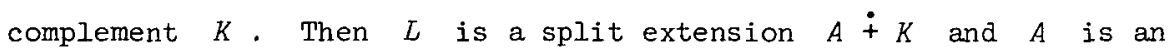
irreducible $K$-module. Our main concern in this section will be to prove conjugacy, under a particular group of automorphisms of $L$, of all complements to $A$.

Suppose $H$ is any subalgebra of a Lie algebra $M$. We define the core of $H$, written $c(H)$, to be the unique largest ideal of $M$ contained in $H$. If $c(H)=0$ we say that $H$ is core-free. We shall be particularly interested in the above configuration in the case when $K$ is core-free. Since $C_{K}(A)$ is an ideal of $L$ (it is centralized by $A$ and idealized by $K$ ) it follows that either (the trivial case) $[A, K]=0$ and $\operatorname{dim} A \leq 1$, or that $A$ is a faithful $K$-module.

It should be noted that $K$, whether core-free or not, is a maximal subalgebra of $L$, for if $X \geq K$ then $0 \neq X \cap A$ is an ideal of $L$, so must be $A$; hence $X=L$.

Suppose that $J$ is also a complement to $A$ in $L$, so that $L=A+J$. For each $k \in K$ there is a unique decomposition 


$$
k=a_{k}+j_{k}
$$

where $a_{k} \in A, j_{k} \in J$. It is easy to verify that the map $k+j_{k}$ is an isomorphism $K \rightarrow J$, which extends to an automorphism $\alpha$ of $L$ if we define

$$
a^{\alpha}=a \quad(a \in A),
$$$$
k^{\alpha}=j_{k} \quad(k \in K)
$$

Hence tririally all complements to $A$ are conjugate under aut $(L)$. However, this is not sufficient for the applications we have in mind, and a stronger theorem must be proved.

Each element $a \in A$ gives rise to an automorphism

$$
\exp \left(a^{*}\right)=1+a^{*}
$$

of $L$, where $a^{*}$ is the adjoint map defined by $x a^{*}=[x, a](x \in L)$. (See, for example, Jacobson [6], p. 9.) The set of such automorphisms, for all $a \in A$, forms a group which we denote by $A(L)$. Our aim is to prove $A(L)$-conjugacy of complements.

THEOREM 2.1. With the above notation, suppose that $K$ acts faithfully on $A$ and that $K$ possesses a 1-dimensional ideal. Then all complements to $A$ are $A(L)$-conjugate.

Proof. Let $X=\langle x\rangle$ be a l-dimensional ideal of $K$ and define $\alpha$ as in $\left(^{*}\right)$. Then $X=\left\langle x^{\alpha}\right\rangle$ is a l-dimensional ideal of $J$. We claim first that $K=I_{L}(X)$, the idealizer of $X$. For suppose $y=a+k \in I_{L}(X), a \in A, k \in K$. Then

$$
[a, x]=[y, x]-[k, x] \in X \cap A=0
$$

so that $[a, x]=0$, or equivalently $[a, X]=0$. However,

$$
B=\{b \in A:[b X]=0\}
$$

is a $K$-module, and since the action is faithful $B=0$. Therefore $a=0$ and $y \in K$.

$$
\text { Hence also } J=K^{\alpha}=\left(I_{L}(X)\right)^{\alpha}=I_{L}\left(X^{\alpha}\right) \text {. }
$$


Now it is clear that $C=[X, A]$ is a $K$-module, and faithfulness now implies that $C \neq 0$, hence $C=A$. Therefore every element of $A$ can be expressed in the form $[x, a]$ for some $a \in A$. Now

$$
x^{\alpha}-x=j_{x}-x=-a_{x} \in A,
$$

so there exists $a \in A$ such that

$$
x^{\alpha}-x=[x, a]
$$

and therefore

$$
x^{\alpha}=x+[x, a]=x\left(1+\dot{a}^{*}\right) .
$$

Let $\beta=1+a^{*} \in \mathrm{A}(L)$. Then

$$
K^{\beta}=\left(I_{L}(X)\right)^{\beta}=I_{L}\left(X^{\beta}\right)=I_{L}\left(X^{\alpha}\right)=J
$$

and the theorem is proved.

Note that this result and its proof are related to theorems of Barnes and Newell [2] and Towers [11]. The underlying idea is of course much older.

The hypotheses of Theorem 2.1 are clearly satisfied if $K$ is nilpotent, or if $K$ is supersoluble (where a supersoluble lie algebra is one with a finite series of ideals with l-dimensional factors, hence in particular is finite-dimensional). By Lie's Theorem (Jacobson [6], p. 52) every finite-dimensional.soluble Lie algebra over an algebraically closed field of characteristic zero is supersoluble. Hence we have the

COROLLARY 2.2. The above theorem holds if either

(i) $K$ is nilpotent,

(ii) $K$ is supersoluble,

(iii) $K$ is finite-dimensional soluble and the underlying field

$\underline{\underline{k}}$ is algebraically closed and of characteristic zero.

Using field-change methods we can prove a certain amount when $K$ is finite-dimensional soluble but $\underline{\underline{k}}$ is not algebraically closed. We still assume $\underline{\underline{k}}$ of characteristic zero. First we must summarize the necessary facts (most of which are to be found in Jacobson [6], p. 295). 
Let $\underline{\underline{K}}$ be a (finite) Galois extension of $\underline{\underline{k}}$, and let $\Gamma$ be the Galois group of $\underline{\underline{K}} / \underline{\underline{k}}$. Let $L$ be a Lie algebra over $\underline{\underline{k}}$, and put

$$
\bar{L}=\underline{\underline{K}} Q_{\underline{\underline{K}}} L \text {. }
$$

For any $\underline{\underline{k}-s u b s p a c e ~} V$ of $L$ put

$$
\bar{V}=\underline{\underline{K}} \underline{\underline{\underline{K}}}_{\underline{\underline{K}}} V \text {. }
$$

We identify $L$ with the $\underline{\underline{k}}$-subspace $\left\{\underline{1}_{\underline{\underline{K}}} \otimes_{\underline{\underline{K}}} x: x \in L\right\}$ of $\bar{L}$. The group $\Gamma$ acts by semilinear transformations of $\bar{L}$ if we define, for $x \in L$, $\lambda \in \underline{\underline{K}}, \gamma \in \Gamma$,

$$
(\lambda \otimes x)^{\gamma}=\lambda^{\gamma} \otimes x
$$

and extend by k-linearity. The set of elements of $\bar{L}$ fixed by every element of $\Gamma$ is precisely $L$. Further if $V$ is any k-subspace of $L$ then $\bar{V}=\bar{V}$ for every $\gamma \in \Gamma$. It is easy to verify that for $x, y \in \bar{L}$ we have

$$
[x, y]^{\gamma}=\left[x^{\gamma}, y^{\gamma}\right]
$$

so in particular if $y \in L$ then

$$
[x, y]^{\gamma}=\left[x^{\gamma}, y\right]
$$

For each $x \in \bar{L}$ we define

$$
\hat{x}=\frac{1}{|\Gamma|} \sum_{\gamma \in \Gamma} x^{\gamma} .
$$

This is fixed by every element of $\Gamma$, so lies in $L$; and we have a k-linear map

$$
\wedge: \bar{L} \rightarrow L
$$

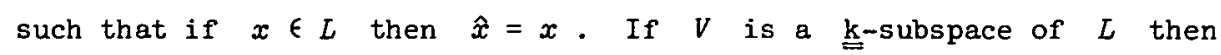
$\hat{\bar{V}}=\bar{V} \cap L=V$.

Now let $K$ be a Lie algebra over $\underline{\underline{k}}$, and $A$ a $K$-module. We say that $A$ is strictly irreducible if for every finite extension $\underline{\underline{K}}$ of $K$, the $\underline{\underline{K}} \underline{\underline{\underline{k}}}_{\underline{\underline{K}}} K$-module $\underline{\underline{K}} \underline{\underline{\underline{K}}} A$ is irreducible.

THEOREM 2.3. Let $L=A \ddagger K$ as above, where $K$ is finite- 
dimensional soluble over a field $\underline{\underline{\mathrm{k}}}$ of characteristic zero, and $A$ is a faithful strictly irreducible K-module. Then all complements to $A$ in $I$ are $\mathrm{A}(L)$-conjugate.

Proof. Over the algebraic closure $\underline{\underline{\mathrm{k}}}^{*}$ of $\underline{\underline{\mathrm{k}}}$ we can make $K$ supersoluble. Hence there is some finite extension $\underline{\underline{K}}$ of $\underline{\underline{k}}$ such that $\bar{K}$ is supersoluble. By passing to a normal closure if necessary we can assume $\underline{\underline{K}} / \underline{\underline{k}}$ normal, and it is separable since the characteristic is zero. Hence $\underline{\underline{K}} / \underline{\underline{k}}$ is a Galois extension, and the above considerations apply. We have $\bar{L}=\bar{A}+\bar{K}$ where (by hypothesis) $\bar{A}$ is an irreducible $\bar{K}$-module.

First let us establish that $\bar{A}$ remains faithful as a $\bar{K}$-module. Suppose $0 \neq C_{\bar{K}}(\bar{A})$, and pick $0 \neq x$ from it. Then $\lambda x$ annihilates $\bar{A}$ for all $\lambda \in \underline{\underline{K}}$. It follows that $\lambda x$ annihilates $A$, so must be zero. But it is easy to see that some choice of $\lambda \in \underline{\underline{K}}$ makes $\lambda x \neq 0$ (say, by taking a basis in $L$ and looking at the expression of $x$ relative to that basis: if $x=\sum \mu_{i} e_{i}$ for $e_{i} \in L, \mu_{i} \in \underline{\underline{K}}$, and some $\mu_{j} \neq 0$, then $\mu_{j}^{-1} x$ has non-zero coefficient for $\left.e_{j}\right)$. This is a contradiction.

Now let $J$ be another complement to $A$ in $L$. Then $\bar{K}$ and $\bar{J}$ are complements to $\bar{A}$ in $\bar{L}$, and by Theorem 2.1 there exists $a \in \bar{A}$ such that $\bar{K}\left(1+a^{*}\right)=\bar{J}$. Hence for each $k \in \bar{K}$ we have $k+[k, a] \in \bar{J}$. Let $k \in K$. Then

$$
\begin{aligned}
k+[k, \hat{a}] & =k+\frac{1}{|\Gamma|} \sum_{\gamma \in \Gamma}\left[k, a^{\gamma}\right] \\
& =(k+[k, a])^{\wedge} \\
& \in \bar{J} \cap L=J .
\end{aligned}
$$

Therefore if we let $\beta=1+\hat{a}^{*} \in A(L)$, then $K^{\beta} \leq J$. Since $K$ is maximal, we have $K^{\beta}=J$ as claimed.

Note that the module $P$ in Hartley's example [5] is strictly irreducible, so such modules do exist.

\section{Finite-dimensional maximal subalgebras}

Suppose that $L$ is a soluble Lie algebra, having a core-free maximal subalgebra $M$. The penultimate term of the derived series of $L$ is a 
non-zero abelian ideal, $A$ say, and $A \ddagger M$ since $M$ is core-free. Therefore by maximality $L=A+M$. Now $A \cap M$ is idealized by $M$ since $A<L$, and by $A$ since $A$ is abelian; hence $A \cap M<L$ and so again $A \cap M=0$. If $0 \neq B$ is an $M$-submodule of $A$, then $B+M=L$ by maximality, and it easily follows that $B=A$. Hence $A$ is irreducible. As remarked earlier, since $M$ is core-free either $M$ acts faithfully on $A$ or $M$ annihilates $A$ and $\operatorname{dim} A=1$.

If $M$ is abelian and finite-dimensional, or if $M$ is finitedimensional and $\operatorname{char}(\underline{\underline{k}})>0$, then every irreducible $M$-module has finite dimension (Amayo and Stewart [1], 11.3.6, Curtis [3], p. 952) and hence $L$ is finite-dimensional. Hence we have proved:

THEOREM 3.1. If $L$ is an infinite-dimensional Lie algebra having a finite-dimensional maximal subalgebra $M$ then:

(i) $L / C(M)$ is a split extension of an abelian ideal $A / C(M)$ by $M / C(M)$, and $A / C(M)$ is a faithful irreducible $M / c(M)$-moduze;

(ii) $\operatorname{char}(\underline{\underline{\mathrm{k}}})=0$, where $\mathrm{k}$ is the underlying field;

(iii) $M / c(M)$ is not abelian.

It is also clear that if $L$ has the structure described in (i) then $M$ is maximal in $L$.

COROLLARY 3.2. If $L$ is a soluble lie algebra having a maximal subalgebra $M$ satisfying $\max -\triangleleft(\min -\triangleleft)$ then $L$ satisfies max- $\triangleleft$ $(\min -\triangleleft)$.

Proof. $L$ has a series of $L$-submodules

$$
0 \leq c(M) \leq A \leq L
$$

and each factor satisfies the maximal (minimal) condition for submodules: $c(M)$ since $M \in \max -\triangleleft(\min -\triangleleft), A / C(M)$ since it is irreducible, $L / A$ since this is isomorphic to $M / C(M)$. Hence by [1], 1.7.4, $L$ satisfies the maximal (minimal) condition for $L$-submodules; that is, max- 4 $(\min -\triangleleft)$.

Recall that for any Lie algebra $L$ the Fitting radical $V(L)$ is the sum of the nilpotent ideals. If $n \in V(L)$ we can define 


$$
\exp \left(n^{*}\right)=1+n^{*}+\frac{1}{2 !} n^{*^{2}}+\ldots
$$

provided $\operatorname{char}(\underline{\underline{\mathrm{k}}})=0$, and this is an automorphism of $L$. We let $N(L)$ be the group generated by all such automorphisms (which is in fact the set of such automorphisms by the Campbell-Hausdorff formula, Jacobson [6], p. 173). We now have a conjugacy theorem:

THEOREM 3.3. Let $L$ be an infinite-dimensional soluble lie algebra having a finite-dimensional maximal subalgebra $M$. If either

(i) $\mathrm{k}$ is algebraically closed, or

(ii) $M / c(M)$ has a l-dimensional ideal,

then all finite-dimensional maximal subalgebras of $L$ are conjugate under $N(L)$.

Proof. Modulo $c(M)$ we are in the situation discussed in Theorem 2.1, by Theorem 3.1. Hence, with $A$ defined as in Theorem 3.1, given any maximal subalgebra $M^{\prime}$ of $L$ of finite dimension, there exists $a \in A$ such that

$$
M\left(1+a^{*}\right) \leq M^{\prime}+c(M) .
$$

(It is easy to see that $\left(M^{\prime}+c(M)\right) / c(M)$ is another complement to $A / C(M)$.) Let $N=V(L)$. If $N$ were finite-dimensional, then $L$ would be finite-dimensional (see [10], p. 335), which is not the case. Then $N+c(M)$ is an ideal of $L$ properly containing $c(M)$. It is easy to see that $A / C(M)$ is the unique minimal ideal of $L / C(M)$, and is contained in every non-zero ideal of $L / C(M)$, so that $A \leq N+c(M)$. Therefore we have

$$
a=n+c
$$

where $n \in N, c \in c(M)$. Furthermore, $M^{\prime}+c(M)$ is finite-dimensional, so a proper subalgebra of $L$, and hence equal to $M^{\prime}$ by maximality. There fore

$$
M \cdot \exp \left(n^{*}\right) \leq M^{\prime}+c(M)=M^{\prime}
$$

and since $\exp \left(n^{*}\right)$ is an automorphism and $M$ is maximal, we have

$$
M \cdot \exp \left(n^{*}\right)=M^{\prime}
$$

and the theorem is proved. 
Note that (ii) will certainly be true if $M$ (or $M / C(M)$ ) is nilpotent (or supersoluble).

Using Theorem 2.3 we can obtain the following version of Theorem 3.3 in the non-algebraically closed case:

THEOREM 3.4. Let $L$ be an infinite-dimensional soluble Lie algebra over $\underline{\underline{\mathrm{k}}}$ having a finite-dimensional maximal subalgebra $M$. Let $\underline{\mathrm{K}}$ be a finite normal extension of $\underline{\underline{k}}$, such that $\underline{\underline{K}}{\underline{\underline{Q_{k}}}}_{\underline{\underline{k}}} M$ is supersoluble. If $\underline{\underline{K}} M$ is a maximal subalgebra of $\underline{\underline{K}} \otimes_{\underline{\underline{K}}} L$ then all finite-dimensional maximal subalgebras of $L$ are conjugate under $N(L)$.

Proof. Follow the proof of Theorem 3.3, tensoring everything with $\underline{\underline{K}}$. The hypothesis that $\underline{\underline{K}} \otimes_{\underline{\underline{K}}} M$ remains maximal ensures that $\underline{\underline{K}} \underline{\underline{K}}_{=} A$ remains irreducible. The proof of Theorem 2.3 yields the required element $a \in A$, and the rest of the proof is exactly as in Theorem 3.3.

Note that in fact the maximality of $M$ in $L$ is a consequence of the maximality of $\underline{\underline{K}} \underline{\underline{\underline{K}}} M$ in $\underline{\underline{K}} \underline{\underline{\underline{K}}}_{\underline{\underline{K}}} L$, and similarly the finiteness of dimension of $M$ follows from that of $\underline{\underline{K}} Q_{\underline{K}} M$. So the force of Theorem 3.4 is that if tensoring with $\underline{\underline{K}}$ leads to a situation where the conjugacy theorem is true, then it remains true in the original situation.

\section{Further remarks}

Let us say that a subalgebra $H$ of a Lie algebra $L$ is (n-step) submaximal if

$$
H=H_{0} \leq H_{1} \leq \ldots \leq H_{n}=L
$$

where $H_{i}$ is a maximal subalgebra of $H_{i+1}$ for $i=0, \ldots, n-1$. As a convention, $L$ is 0 -step submaximal in $L$. If we impose finiteness conditions on a submaximal subalgebra of $L$ then this can have strong consequences for $L$, as is clear from an induction argument based on Corollary 3.2. In particular if $H$ is submaximal in $L$, where $L$ is soluble, and if $H$ satisfies $\max -\triangleleft(\min -\triangleleft)$ then so does $L$. If $H$ is finite-dimensional, and either $\operatorname{char}(\underline{\underline{k}})>0$ or $H$ is abelian, then $L$ is finite-dimensional. We can also phrase this in the language of closure operations. For any class $\underline{\underline{X}}$ of Lie algebras we define $\underline{\underline{\underline{X}}}$ to be the 
class of Lie algebras having a submaximal $\underline{\underline{X}}$-subalgebra. It is easy to verify that $M$ is a closure operation (except that $m(0)$ is the class of algebras of dimension $\leq 1$, which makes little difference: we can ignore. it, or redefine $m(0)=(0))$ : the above remarks take the form that (relative to the class of soluble algebras) max- $\triangleleft$, min- $\triangleleft$ are $m-c l o s e d$, and that the class $\underline{\underline{F}}$ of finite-dimensional algebras is $M$-closed in positive characteristic.

One might enquire about analogous theorems for groups. It is fairly clear that Theorem 3.1 ( $i$ ) carries over, and hence the relative $M$-closure . of $\max -\triangleleft$ and $\min -\triangleleft$. However, no satisfactory analogue of Theorem 3.3 exists, for the following reason. The group-theoretic analogue of a finite-dimensional soluble Lie algebra lies somewhere between two extremes: finite soluble groups and polycyclic groups. However, irreducible modules for such groups are of necessity finite. (For finite groups this follows from results of Hall [4]; for polycyclic groups it is a deep recent result of Roseblade [7,8].) Hence both the class of finite soluble groups and the class of polycyclic groups are m-closed (relative to the class of soluble groups) and so no analogue of the configuration of Theorem 3.3 can be found.

We conclude with an example to illustrate the possibilities in the non-soluble case. Let $\underline{\underline{k}}$ be any field of characteristic zero, and let $W$ have basis $w_{i}(i \in Z)$ and multiplication

$$
\left[w_{i}, w_{j}\right]=(i-j) w_{i+j}
$$

Then $W$ is simple (see [1], Chapter 9). If we put

$$
\begin{aligned}
& L=\left\langle w_{-1}, w_{0}, w_{1}, w_{2}, \ldots\right\rangle, \\
& M=\left\langle w_{-1}, w_{0}, w_{1}\right\rangle
\end{aligned}
$$

then the methods and results of [1], Chapter 9, show (the exact details hardly matter) that $M$ and $L$ are simple, $M$ is maximal in $L$, and $M$ and $L$ are simple. In fact, $L / M$ is an irreducible $M$-module. Since $L$ is simple it is not any sort of split extension. Further, $M$ is the unique finite-dimensional maximal subalgebra of $L$, apart from possibly 1-dimensional subalgebras. Whether any 1-dimensional maximal subalgebras of $L$ exist is a complicated combinatorial question which we leave open. 
If they do, then no conjugacy theorem is possible. If not, then $M$ is the unique maximal finite-dimensional subalgebra of $L$, whereas such behaviour is impossible in the soluble case.

\section{References}

[1] R.K. Amayo and I.N. Stewart, Infinite-dimensional Lie algebras (Noordhoff, Leyden, to appear).

[2] Donald W. Barnes and Martin L. Newell, "Some theorems on saturated homomorphs of soluble Lie algebras", Math. 2. 115 (1970), $179-187$.

[3] Charles W. Curtis, "Noncommutative extensions of Hilbert rings", Proc. Amer. Math. Soc. 4 (1953), 945-955.

[4] P. Hall, "On the finiteness of certain soluble groups", Proc. London Math. Soc. (3) 9 (1959), 595-622.

[5] B. Hartley, "Locally nilpotent ideals of a Lie algebra", Proc. Combridge Phizos. Soc. 63 (1967), 257-272.

[6] Nathan Jacobson, Lie algebras (Interscience [John Wiley \& Sons], New York, London, 1962).

[7] J.E. Roseblade, "Polycyclic group rings and the Nullstellensatz", Conference on group theory, University of Wisconsin-Parkside 1972, 156-167 (Lecture Notes in Mathematics, 319. SpringerVerlag, Berlin, Heidelberg, New York, 1973).

[8] J.E. Roseblade, "Group rings of polycyclic groups", J. Pure Appl. Algebra 3 (1973), 307-328.

[9] I.N. Stewart, "An algebraic treatment of Mal'cev's theorems concerning nilpotent Lie groups and their Lie algebras", Compositio Math. 22 (1970), 289-312.

[10] I.N. Stewart, "A property of locally finite Lie algebras", J. London Math. Soc. (2) 3 (1971), 334-340. 
[11] D.A. Towers, "A Frattini theory for algebras", PhD thesis, University of Leeds, 1972.

Mathematisches Institut der Universität, Tübingen,

Germany. 